\title{
Glutathione and immune function
}

\author{
Wulf Dröge* and Raoul Breitkreutz \\ Department of Immunochemistry, Deutsches Krebsforschungszentrum, Im Neuenheimer Feld 280, \\ D-69120 Heidelberg, Germany
}

\begin{abstract}
The immune system works best if the lymphoid cells have a delicately balanced intermediate level of glutathione. Even moderate changes in the intracellular glutathione level have profound effects on lymphocyte functions. Certain functions, such as the DNA synthetic response, are exquisitely sensitive to reactive oxygen intermediates and, therefore, are favoured by high levels of the antioxidant glutathione. Certain signal pathways, in contrast, are enhanced by oxidative conditions and favoured by low intracellular glutathione levels. The available evidence suggests that the lymphocytes from healthy human subjects have, on average, an optimal glutathione level. There is no indication that immunological functions such as resistance to infection or the response to vaccination may be enhanced in healthy human subjects by administration of glutathione or its precursor amino acid cysteine. However, immunological functions in diseases that are associated with a cysteine and glutathione deficiency may be significantly enhanced and potentially restored by cysteine supplementation. This factor has been studied most extensively in the case of human immunodeficiency virus (HIV)-infected patients who were found to experience, on average, a massive loss of $\mathrm{S}$ equivalent to a net loss of approximately $4 \mathrm{~g}$ cysteine/d. Two randomized placebo-controlled trials have shown that treatment of HIV-infected patients with $\mathrm{N}$-acetylcysteine caused in both cases a significant increase in all immunological functions under test, including an almost complete restoration of natural killer cell activity. It remains to be tested whether cysteine supplementation may be useful also in other diseases and conditions that are associated with a low mean plasma cystine level and impaired immunological functions.
\end{abstract}

Glutathione: Immunity: Cysteine

\section{The importance of glutathione and cysteine for lymphocyte functions in vitro}

Studies of lymphocyte functions in cell cultures have been greatly facilitated by the empirical finding that these functions are strongly enhanced by thiol compounds (Meister \& Anderson, 1983; Ishii et al. 1987). This finding is most strikingly exemplified by the fact that immunologists have been adding 2-mercaptoethanol routinely to the cell culture medium when studying immunological responses of murine lymphocytes (Fanger et al. 1970). Many years after these first experiments it became clear that 2-mercaptoethanol enhances the cysteine supply to the lymphoid cells (Ishii et al. 1987), and thereby increases the intracellular level of the cysteine-containing tripeptide glutathione (see Fig. 1; Meister, 1983; Meister \& Anderson, 1983).

\section{Need for a delicately balanced intermediate level of glutathione}

As the quantitatively most important intracellular antioxidant, and as a substrate for the GSH peroxidase reaction, glutathione serves as the major scavenger of reactive oxygen species. Certain lymphocyte functions, such as the DNA synthetic response, are exquisitely sensitive to reactive oxygen species and, therefore, are favoured by relatively high levels of glutathione. Even a moderate depletion of the intracellular glutathione pool by treatment with buthionine sulfoximine, a specific inhibitor of GSH biosynthesis, has dramatic consequences for a variety of lymphocyte 


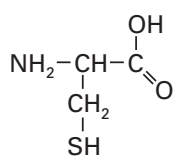

Cysteine

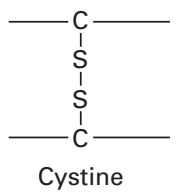

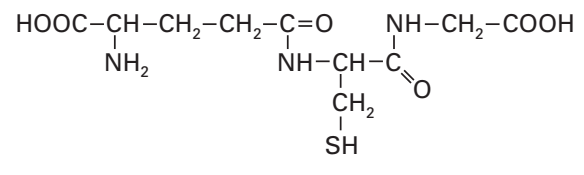

Glutathione

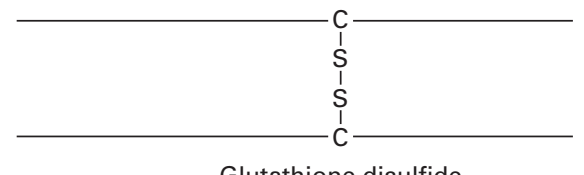

Glutathione disulfide
Fig. 1. The most important sulfhydryl compounds.

functions (Hamilos \& Wedner, 1985; Beutler, 1989; Gmünder et al. 1990a,b; Gmünder \& Dröge, 1991; Dröge et al. 1994). Interleukin 2-dependent functions, including $\mathrm{T}$-cell proliferation, the generation of $\mathrm{CD}^{+} \mathrm{T}$-cell blasts, cytotoxic T-cell activity, lymphokine-activated killer cells and natural killer cells, are particularly sensitive to glutathione depletion (for review, see Dröge et al. 1994). Other types of lymphocyte responses, including the production of $\gamma$-interferon and interleukin 2 and the expression of their respective mRNA species, are either unaffected or even enhanced by moderate concentrations of reactive oxygen species and favoured by a decrease in the intracellular glutathione level (Roth \& Dröge, 1987, 1991; Los et al. 1995). There is now a substantial body of evidence indicating that certain signal cascades in $\mathrm{T}$ lymphocytes can be amplified by a mild oxidation of the glutathione pool (Mihm et al. 1991; Schreck et al. 1991; Meyer et al. 1993; Galter et al. 1994; Schenk et al. 1994; Nakamura et al. 1997; Adler et al. 1999). Immunological reactions of lymphocytes in vitro, therefore, may be impaired not only by glutathione depletion or by the absence of 2-mercaptoethanol from the culture medium, but also by superoptimal concentrations of 2-mercaptoethanol, the optimal concentration being approximately $30 \mu \mathrm{M}$ (for review, see Dröge et al. 1994).

The need for a balanced intermediate level of glutathione appears to exist also in the intact organism. A study of eighty-five untreated healthy human subjects revealed a mean intracellular glutathione level of 25.5 (SE 1.0) nmol/ mg protein (Kinscherf et al. 1994). Individuals with intermediate levels of glutathione $(20-30 \mathrm{nmol} / \mathrm{mg}$ protein) were found to have, on average, a significantly higher number of $\mathrm{CD}^{+}{ }^{+} \mathrm{T}$-cells than individuals with either lower or higher intracellular glutathione levels. This finding indicated that (1) the immune system may be exquisitely sensitive not only to a glutathione deficiency, but also to an excess of glutathione, and (2) the mean glutathione level of healthy human subjects is approximately optimal. Individuals who during a 4-week observation period with episodes of intense anaerobic physical exercise moved from the optimal to the suboptimal range of glutathione (10-20 nmol/mg) experienced, on average, a $30 \%$ decrease in $\mathrm{CD}^{+}{ }^{+} \mathrm{T}$-cell numbers (Kinscherf et al. 1994). This decrease was prevented by treatment with $\mathrm{N}$-acetyl-cysteine, a putative precursor of glutathione biosynthesis. However, treatment with $\mathrm{N}$-acetyl-cysteine had beneficial effects only on that group of individuals who happened to experience a period with abnormally low intracellular glutathione levels. Importantly, in this case $\mathrm{N}$-acetyl-cysteine caused the relative increase in $\mathrm{CD}_{4}{ }^{+} \mathrm{T}$-cell numbers despite decreasing glutathione levels, and not by increasing the glutathione level (Kinscherf et al. 1994). In another study (Breithaupt et al. 1996) it was shown that the decreased mitogenic responses and decreased synthesis of interleukin 2 in $\mathrm{CD}^{+}$ and $\mathrm{CD}^{+}$T-cells from aged rhesus monkeys (Macaca mulatta) were reversed by treatment with the glutathione precursor $\mathrm{N}$-acetyl-cysteine.

Antigen-presenting cells such as macrophages release substantial amounts of cysteine that raise the intracellular glutathione level of activated T-cells in their vicinity (Gmünder et al. 1990a). This cysteine-delivering function appears to be physiologically important for the T-cell response, because the early phase of blast transformation has a particularly high demand for cysteine (Roth \& Dröge, 1994). Studies of cytotoxic and protective responses against a series of tumour cells and tumour cell variants in vivo (Lim et al. 1992) indicated that the capacity of the stimulator cells to release cysteine into the extracellular space is indeed one of the limiting factors that determines their immunogenic properties if administered in vivo in relatively large numbers. Cells that fail to release large amounts of cysteine can, nevertheless, be immunogenic in vivo and in vitro, if they are administered in relatively small numbers (Roth \& Dröge, 1994). Under these conditions, exogenous glutathione may even inhibit T-cell responses. In contrast, if administered in high numbers these 'non-professional' stimulator cells induce, in the absence of exogenous glutathione, an almost complete inhibition of the T-cell response. Taken together, there is now a large amount of information indicating that the administration of cysteine as a glutathione precursor is not a simple routine path to immunopotentiation in the healthy intact organism, but may be useful only if the responding T-cells have, for one reason or another, an abnormally low glutathione level. The administration of glutathione or cysteine as a strategy to enhance resistance to infection or to improve vaccination programmes is not warranted unless there is diagnostic evidence for a cysteine or glutathione deficiency.

\section{Massive loss of cysteine in human immunodeficiency virus infection as a pathogenetic factor}

The best studied case of a cysteine and glutathione deficiency is human immunodeficiency virus (HIV) infection. HIV-positive individuals have, in general, abnormally low cyst(e)ine and glutathione levels (Dröge et al. 1988; Eck et al. 1989). To ameliorate these changes and their immunological consequences, we (Dröge, 1989; Dröge et al. 1992) proposed treatment with a cysteine derivative such as $\mathrm{N}$-acetyl-cysteine. Subsequently, the abnormal cysteine status of HIV-infected patients and simian immunodeficiency virus-infected macaques has been confirmed by other research groups (Eck et al. 1991; Staal et al. 1992; Hortin et al. 1994; Akerlund et al. 1996; Hack et al. 1997; Walmsley et al. 1997). Several groups also reported a significant decrease in the intracellular glutathione level of the peripheral-blood mononuclear cells from HIV-infected patients (Eck et al. 1989; Roederer et al. 
1991; De Quay et al. 1992; Herzenberg et al. 1997; Jahoor et al. 1999) and simian immunodeficiency virus-infected rhesus macaques (Eck et al. 1991). Buhl et al. (1989) and Pacht et al. (1997) demonstrated abnormally-low glutathione levels in the blood plasma and alveolar lining fluid of HIV-infected individuals. N-acetyl-cysteine eventually became a widely used drug for HIV-positive patients in the USA and Western Europe.

The magnitude of the daily loss of S amino acids in HIV infection, however, has been determined only recently by two independent strategies. Since the massive cysteine catabolism in HIV-infected patients yields sulfate (i.e. the salt of sulfuric acid), amongst other products, the net loss can be demonstrated most easily by the sulfate content of the urine (Breitkreutz et al. 2000a). In addition, it has been shown that the peripheral tissue of the lower extremities of HIV-infected patients (i.e. mainly the skeletal muscle tissue), in contrast to the tissue of healthy human subjects, releases substantial amounts of sulfate, indicating that the skeletal muscle tissue is the site of elevated cysteine catabolism (Breitkreutz et al. 2000a). From the arteriovenous differences of plasma amino acid and sulfate concentrations of HIV-infected patients and healthy subjects it was estimated that the skeletal muscle tissue of an HIV-positive individual with a body weight of approximately $70 \mathrm{~kg}$ produces, on average, an excessive amount of sulfate, equivalent to a daily catabolism of more than $5 \mathrm{~g}$ cysteine/d (Breitkreutz et al. 2000a). These findings were in agreement with earlier studies on simian immunodeficiency virus-infected rhesus macaques, showing that intracellular sulfate levels in the skeletal muscle tissue are markedly increased and the intracellular glutathione levels correspondingly decreased (Gross et al. 1996). Since submission of the publication by Breitkreutz et al. (2000a), the massive loss of urinary $S$ has been confirmed in additional groups of patients. The results are shown in Table 1. Among healthy control persons, the daily sulfate excretion showed only a very small variability, whereas the sulfate excretion amongst HIV-infected individuals showed for unknown reasons a strong inter-individual and intraindividual variability. Due to this variability, the mean value for daily sulfate excretion amongst asymptomatic patients in Table 1 differs slightly from the published data of Breitkreutz et al. (2000a), but in principle confirms the earlier findings. Since there is a small variability in the sulfate excretion amongst healthy individuals, the increase in sulfate excretion of HIV-infected patients is significant $(P<0 \cdot 01)$ despite its large variability. Considering the

Table 1. Loss of urinary sulfate in asymptomatic human immunodeficiency virus-positive $\left(\mathrm{HIV}^{+}\right)$patients (Mean values with their standard errors)

\begin{tabular}{lccc}
\hline & & \multicolumn{2}{c}{ Urinary sulfate $(\mathrm{g} / \mathrm{d})$} \\
\cline { 3 - 4 } & $n$ & Mean & SE \\
\hline Healthy subjects & 38 & 1.88 & 0.13 \\
HIV+ $^{+}$without ART & 16 & 4.80 & 0.84 \\
HIV+ $^{+}$with HAART & 21 & 3.89 & 0.60
\end{tabular}

ART, anti-retroviral therapy; HAART, highly-active anti-retroviral therapy including at least one protease inhibitor. normal excretion of $1.8 \mathrm{~g}$ sulfate is usually balanced by the dietary protein, the mean loss of $4.8 \mathrm{~g}$ sulfate would be equivalent to a net loss of $3 \mathrm{~g}$ sulfate or approximately $4 \mathrm{~g}$ cysteine/d in asymptomatic HIV patients. Although the increase in the sulfate excretion of HIV-infected patients is statistically significant, the mean value of $4 \mathrm{~g}$ cysteine/d should only be taken as a relatively imprecise estimate of the mean value for the total asymptomatic HIV-infected population, in view of the relatively small number of sixteen HIV-infected individuals tested so far (Table 1). Nevertheless, the results obtained with these sixteen HIV-infected patients showed that this loss of S can be surprisingly high and, generally, would not necessarily be compensated by an increase in dietary protein. Even if the longitudinal mean loss of $\mathrm{S}$ of an individual patient is not $4 \mathrm{~g} / \mathrm{d}$, but only 3 or $2 \mathrm{~g} / \mathrm{d}$, it is reasonable to assume that this daily loss would be large enough to cause serious health problems within a few years. This conclusion is based, amongst other arguments, on the known fact that a protein deficiency may lead to a life-threatening condition within a short period of time, even amongst otherwise-healthy subjects. In several earlier studies on protein-deficient diets in experimental animals (Table 2), the S-containing amino acids cysteine and its precursor methionine were identified as the most-limiting amino acids. In view of these facts, there is a strong possibility that the virus-induced cysteine deficiency may indeed be one of the causative factors leading to disease progression, and may represent a central problem in the pathogenetic mechanism.

It was also important to note in this context that the urinary excretion of sulfate in the early asymptomatic $\mathrm{HIV}$-infected individuals was elevated much more markedly than that of urea, indicating that the excessive cysteine catabolism resulted mainly from a net loss of glutathione (i.e. from a substance with a relatively high $\mathrm{S}: \mathrm{N}$ value) and not from net protein catabolism. At face value this cysteine deficiency may be reminiscent of the various other deficiencies that have been reported for HIV-infected patients. However, most of these deficiencies have been observed only in the late stages, as a consequence of the advanced disease condition. In the late stages of the disease a net protein catabolism is also a common finding. The massive cysteine catabolism, in contrast, is already demonstrable in the early asymptomatic stage of the infection and, therefore, is possibly a causative factor for the subsequent disease progression.

In view of these considerations, it could be important for both the patients and the physicians to know that the

Table 2. Identification of cysteine and the cysteine precursor methionine as the most-limiting amino acid in studies on protein-deficient diets

\begin{tabular}{ll}
\hline Animal & Reference \\
\hline Dogs & Allison et al. (1947) \\
Pigs & Lubaszewska et al. (1973) \\
Rats & Lubaszewska et al. (1973) \\
& Yoshida \& Moritoki (1974) \\
Chickens & Okumura \& Muramatsu (1978) \\
& Webel \& Baker (1999)
\end{tabular}


massive loss of $\mathrm{S}$ is not prevented by treatment with a combination therapy, including at least one protease inhibitor (Table 1). The analysis of twenty-one patients who have been treated with a combination therapy, including at least one protease inhibitor (mainly Viracept or Crixivan), for at least 6 months revealed a daily mean loss of $S$ of approximately $4 \mathrm{~g} / \mathrm{d}$.

The causative role of this cysteine deficiency in the development of the immunological dysfunctions in HIV infection has been demonstrated in two randomized placebo-controlled studies on the therapeutic effects of $\mathrm{N}$-acetyl-cysteine in two groups of asymptomatic HIV-infected patients with and without anti-retroviral therapy respectively. Both studies consistently showed that treatment of these patients with the additional source of cysteine caused a significant enhancement of several immunological functions under test, including restoration of natural killer cell activity to almost normal levels (Breitkreutz et al. 2000b). Currently, there are no follow-up data available. It is important to note, however, that the restoration of the immune system is a widely accepted aim in the therapy for HIV infection. Together with the demonstration of the massive loss of $\mathrm{S}$, the immune restoration achieved with $\mathrm{N}$-acetyl-cysteine should be sufficient reason to establish cysteine supplementation as a standard therapy complementary to anti-retroviral therapy in HIV infection. The results of several earlier studies by various laboratories (for review, see Dröge \& Breitkreutz, 1999) have already provided evidence suggesting that $\mathrm{N}$-acetyl-cysteine may be useful in the therapy of HIVinfected patients. Amongst other groups, Herzenberg et al. (1997) reported significantly improved 2-year survival rates amongst HIV-infected patients after treatment with $\mathrm{N}$-acetyl-cysteine. The general acceptance of this report was compromised, however, by the fact that the study on the 2-year survival rate had not been rigorously randomized. Similar or other problems have also limited the acceptance of the other studies (for review, see Dröge \& Breitkreutz, 1999).

In view of the enormous variability of cysteine catabolism in HIV-infected patients, the individual dose in the two placebo-controlled trials was adjusted according to individual needs (Breitkreutz et al. 2000b). Advantage was taken of the fact that treatment with $\mathrm{N}$-acetyl-cysteine also increases the otherwise abnormally low plasma glutamine levels. The glutamine level was found to be by far the most precise and most useful variable for individual dose adjustment, and as such clearly superior to the intracellular glutathione level of the lymphocytes or the plasma albumin level. The mean plasma glutamine level of healthy human subjects is approximately $600 \mu \mathrm{M}$. We recommend that the level of cysteine supplementation (i.e. the dose of $\mathrm{N}$-acetyl-cysteine) may be chosen initially to be of the order of $2-4 \mathrm{~g} / \mathrm{d}$, and may be decreased if the plasma glutamine level exceeds $700 \mu \mathrm{M}$.

\section{Concluding remarks}

Although HIV infection is the best studied immunodeficiency associated with a cysteine and glutathione deficiency, there are indications that a similar, if less extreme, cysteine deficiency with similar immunological consequences may also occur in other diseases and conditions (for review, see Dröge \& Holm, 1997; see also Table 3). Whenever tested the decrease in plasma cystine levels was associated with a corresponding decrease in plasma glutamine levels, an increase in plasma glutamate, an increased rate of urea production and a more- or lessstriking impairment of immunological functions, including a prominent decrease in natural killer cell activity (Dröge \& Holm, 1997). Whether supplementation of these patients with an additional source of cysteine will also help to improve the immunological functions in these cases remains to be shown.

In HIV infection $\mathrm{N}$-acetyl-cysteine has already been widely used for several years at arbitrarily chosen doses as a supplementary medication in several Western countries. We are now in a much better position, since we have a quantitative estimate of the need for supplementation. The assays for the determination of daily sulfate excretion are relatively simple, and may easily be extended to the other diseases and conditions mentioned earlier. $\mathrm{N}$-acetylcysteine is a relatively inexpensive and safe drug. The data from the two randomized trials on the effect of $\mathrm{N}$-acetylcysteine on several immunological variables (Breitkreutz et al. 2000b), in conjunction with a decreasing enthusiasm for the new generation of anti-retroviral drugs, is expected to increase the acceptance of $\mathrm{N}$-acetyl-cysteine as a standard complementary treatment in HIV infection. Last but not least, the treatment with $\mathrm{N}$-acetyl-cysteine may be useful also for those HIV-infected patients who cannot tolerate or cannot afford to pay for the treatment with anti-retroviral drugs.

Table 3. Diseases and conditions associated with abnormally low plasma cystine $\left(\mathrm{Cys}_{2}\right)$ and glutamine (GIn) levels

\begin{tabular}{|c|c|c|c|c|c|}
\hline Type & $\mathrm{Cys}_{2}$ & Gln & Glu & Urea production & Immune functions $\dagger$ \\
\hline HIV infection, late asymptomatic & $\downarrow \downarrow$ & $\downarrow \downarrow$ & $\uparrow$ & $\uparrow \uparrow$ & $\downarrow \downarrow N K$ etc. \\
\hline Sepsis, major injury and trauma* & $\downarrow \downarrow$ & $\downarrow \downarrow$ & $\uparrow$ & $\uparrow \uparrow$ & $\downarrow \downarrow N K$ etc. \\
\hline Crohn's disease & $\downarrow \downarrow$ & $\downarrow$ & $(\uparrow)$ & $\uparrow \uparrow$ & $\downarrow \downarrow N K$ etc. \\
\hline Ulcerative colitis & $\downarrow \downarrow$ & $\downarrow$ & $(\uparrow)$ & $\uparrow \uparrow$ & $\downarrow \downarrow N K$ etc. \\
\hline Overtraining & nd & $\downarrow \downarrow$ & $\uparrow$ & $\uparrow \uparrow$ & $\downarrow \downarrow$ NK etc., OI \\
\hline Chronic fatigue syndrome & $\downarrow \downarrow$ & $\downarrow \downarrow$ & nd & nd & $\downarrow \downarrow$ NK etc. \\
\hline Starvation & $\downarrow \downarrow$ & nd & nd & $\uparrow \uparrow$ & $\downarrow$ Immunological deficiency OI \\
\hline
\end{tabular}

Glu, glutamate; nd, Not determined or not detected; NK, natural killer; OI, opportunistic infections; $\downarrow$, decrease; $\uparrow$, increase.

${ }^{*}$ Cystine levels are inversely correlated with urea production.

† NK cell activity, or other immunological variables, including CD4+:CD8 ${ }^{+}$T-cell values. 


\section{Acknowledgements}

The assistance of Mrs I. Fryson in the preparation of this manuscript is gratefully acknowledged.

\section{References}

Adler V, Yin Z, Tew KD \& Ronai Z (1999) Role of redox potential and reactive oxygen species in stress signaling. Oncogene 18, 6104-6111.

Akerlund B, Jarstrand C, Lindeke B, Sönnerborg A, Akerblad A-Ac \& Rasool O (1996) Effect of N-acetylcysteine (NAC) treatment on HIV-1 infection: a double-blind placebo-controlled trial. European Journal of Clinical Pharmacology 50, 457-461.

Allison JB, Anderson JA \& Seeley RD (1947) Some effects of methionine on the utilization of nitrogen in the adult dog. Journal of Nutrition 33, 361-370.

Beutler E (1989) Nutritional and metabolic aspects of glutathione. Annual Review of Nutrition 9, 287-302.

Breithaupt T, Eylar EH, Baez I, Vasquez A, Colon-Martinez S, Rodriquez J \& Kesseler M (1996) N-acetylcysteine (NAC) reverses the supposed mitogenic responses of $\mathrm{CD}^{+}$and $\mathrm{CD} 8^{+} \mathrm{T}$ cells from aged rhesus monkeys. FASEB Journal 10, 1849 Abstr.

Breitkreutz R, Holm S, Pittack N, Beichert M, Babylon A, Yodoi J \& Dröge W (2000a) Massive loss of sulphur in HIV infection. AIDS Research and Human Retroviruses 3, 203-209.

Breitkreutz R, Pittak N, Nebe CT, Schuster D, Brust J, Beichert M, Hack V, Daniel V, Edler L \& Dröge W (2000b) Improvement of immune functions in HIV infection by sulfur supplementation two randomized trials. Journal of Molecular Medicine $\mathbf{7 8 ,}$ 55-62.

Buhl R, Holroyd K, Mastrangeli A, Cantin AM, Jaffe HA, Wells FB, Saltini C \& Crystal RG (1989) Systemic glutathione deficiency in symptom-free HIV-seropositive individuals. Lancet ii, 1294-1298.

De Quay B, Malinverni R \& Lauterburg BH (1992) Glutathione depletion in HIV-infected patients: role of cysteine deficiency and effect of oral N-acetylcysteine. AIDS 6, 815-819.

Dröge W (1989) Metabolische Störungen bei HIV-Infektion (Metabolic disturbances with HIV infection). In Project News, no. 2, p. 4. Berlin, Germany: AIDS-Zentrum des Bundesgesundheitsamtes.

Dröge W \& Breitkreutz R (1999) N-acetyl-cysteine in the therapy of HIV-positive patients. Current Opinion in Clinical Nutrition and Metabolic Care 2, 493-498.

Dröge W, Eck H-P \& Mihm S (1992) HIV-induced cysteine deficiency and $\mathrm{T}$ cell dysfunctions - a rationale for treatment with N-acetyl-cysteine. Immunology Today 13, 211-214.

Dröge W, Eck H-P, Näher H, Pekar U \& Daniel V (1988) Abnormal amino acid concentrations in the blood of patients with acquired immune deficiency syndrome (AIDS) may contribute to the immunological defect. Biological Chemistry Hoppe-Seyler 369, 143-148.

Dröge W \& Holm E (1997) Role of cysteine and glutathione in HIV infection and other diseases associated with muscle wasting and immunological dysfunction. FASEB Journal 11, 1077-1089.

Dröge W, Schulze-Osthoff K, Mihm S, Galter D, Schenk H, Eck H-P, Roth S \& Gmünder H (1994) Function of glutathione and glutathione disulfide in immunology and immunopathology. FASEB Journal 8, 1131-1138.

Eck H-P, Gmünder H, Hartmann M, Petzoldt D, Daniel V \& Dröge W (1989) Low concentrations of acid soluble thiol (cysteine) in the blood plasma of HIV-1 infected patients. Biological Chemistry Hoppe-Seyler 370, 101-108.

Eck H-P, Stahl-Hennig H, Hunsmann G \& Dröge W (1991) Metabolic disorder as an early consequence of simian immuno- deficiency virus infection in rhesus monkeys. Lancet 338, 346-347.

Fanger MW, Hart DA, Wells JV \& Nisonoff A (1970) Enhancement by reducing agents of the transformation of human and rabbit peripheral lymphocytes. Journal of Immunology $\mathbf{1 0 5}$, 1043-1045.

Galter D, Mihm S \& Droge W (1994) Distinct effects of glutathione disulphide on the nuclear transcription factor kappa B and the activator protein-1. European Journal of Biochemistry 221, 639-648.

Gmünder H \& Dröge W (1991) Differential effects of glutathione depletion on T cell subsets. Cellular Immunology 138, 229-237.

Gmünder H, Eck H-P, Benninghoff B, Roth S \& Dröge W (1990a) Macrophages regulate intracellular glutathione levels of lymphocytes. Cellular Immunology 129, 32-46.

Gmünder H, Roth S, Eck H-P, Gallas H, Mihm S \& Dröge W (1990b) Interleukin-2 mRNA expression, lymphokine production and DNA synthesis in glutathione-depleted T cells. Cellular Immunology 130, 520-528.

Gross A, Hack V, Stahl-Hennig C \& Dröge W (1996) Elevated hepatic $\gamma$-glutamylcysteine synthetase activity and abnormal sulfate levels in liver and muscle tissue may explain abnormal cysteine and glutathione levels in SIV-infected rhesus macaques. AIDS Research and Human Retroviruses 12, 1639-1641.

Hack V, Schmid D, Breitkreutz R, Stahl-Hennig C, Drings P, Kinscherf R, Taut F, Holm E \& Dröge W (1997) Cystine levels, cystine flux and protein catabolism in cancer cachexia, HIV/SIV infection and senescence. FASEB Journal 11, 84-92.

Hamilos DL \& Wedner HJ (1985) The role of glutathione in lymphocyte activation. I. Comparison of inhibitory effects of buthionine sulfoximine and 2-cyclohexene-1-one by nuclear size transformation. Journal of Immunology 135, 2740-2747.

Herzenberg LA, De Rosa SC, Dubs JG, Roederer M, Anderson MT, Ela SW, Deresinski SC \& Herzenberg LA (1997) Glutathione deficiency is associated with impaired survival in HIV disease. Proceedings of the National Academy of Sciences USA 94, 1967-1972.

Hortin GL, Landt M \& Powderly WG (1994) Changes in plasma amino acid concentrations in response to HIV-1 infection. Clinical Chemistry 40, 785-789.

Ishii T, Sugita Y \& Bannai S (1987) Regulation of glutathione levels in mouse spleen lymphocytes by transport of cysteine. Journal of Cellular Physiology 133, 330-336.

Jahoor F, Jackson A, Gazzard B, Philips G, Sharpstone D, Frazer ME \& Heird W (1999) Erythrocyte glutathione deficiency in symptom-free HIV infection is associated with decreased synthesis rate. American Journal of Physiology 276, E205-E211.

Kinscherf R, Fischbach T, Mihm S, Roth S, Hohenhaus-Sievert E, Weiss C, Edler L, Bärtsch P \& Dröge W (1994) Effect of glutathione depletion and oral $\mathrm{N}$-acetyl-cysteine treatment on $\mathrm{CD}^{+}$and $\mathrm{CD}^{+}$cells. FASEB Journal 8, 448-451.

Lim J-S, Eck H-P, Gmünder H \& Dröge W (1992) Expression of increased immunogenicity by thiol releasing tumor variants. Cellular Immunology 140, 345-356.

Los M, Schenk H, Hexel K, Baeuerle PA, Dröge W \& Schulze-Osthoff K (1995) IL-2 gene expression and NF-kB activation through $\mathrm{CD} 28$ requires reactive oxygen production by 5-lipoxygenase. EMBO Journal 14, 3731-3740.

Lubaszewska S, Pastuszewska B \& Kielanowski J (1973) Effect of methionine supplementation of a protein-free diet on the nitrogen excretion in rats and pigs. Tierphysiologie, Tierernährung und Futtormittelkunde 31, 120-128.

Meister A (1983) Selective modification of glutathione metabolism. Science 220, 471-477.

Meister A \& Anderson ME (1983) Glutathione. Annual Review of Biochemistry 52, 711-760. 
Meyer M, Schreck R \& Baeuerle PA (1993) $\mathrm{H}_{2} \mathrm{O}_{2}$ and antioxidants have opposite effects on activation of NF- $\mathrm{KB}$ and $\mathrm{AP}-1$ in intact cells: AP-1 as secondary antioxidant-responsive factor. $E M B O$ Journal 12, 2005-2015.

Mihm S, Ennen J, Pessara U, Kurth R \& Dröge W (1991) Inhibition of HIV-1 replication and NF- $\kappa$ B activity by cysteine and cysteine derivatives. AIDS 5, 497-503.

Nakamura H, Nakamura K \& Yodoi J (1997) Redox regulation of cellular activation. Annual Review of Immunology 15, 351-369.

Okumura J \& Muramatsu T (1978) Effect of dietary methionine and arginine on the excretion of nitrogen in cocks fed on a proteinfree diet. Japanese Poultry Science 15, 69-73.

Pacht ER, Diaz P, Clanton T, Hart J \& Gadek JE (1997) Alveolar fluid glutathione decreases in asymptomatic HIV-seropositive subjects over time. Chest 112, 785-788.

Roederer M, Staal FJT, Osada H \& Herzenberg LA (1991) CD4 and CD8 $\mathrm{T}$ cells with high intracellular glutathione levels are selectively lost as HIV infection progresses. International Immunology 3, 933-937.

Roth S \& Dröge W (1987) Regulation of T cell activation and T cell growth factor (TCGF) production by hydrogen peroxide. Cellular Immunology 108, 417-424.

Roth S \& Dröge W (1991) Regulation of interleukin-2 production, interleukin 2 mRNA expression and intracellular glutathione levels in ex vivo derived T lymphocytes by lactate. European Journal of Immunology 21, 1933-1937.
Roth S \& Dröge W (1994) Glutathione reverses the inhibition of $\mathrm{T}$ cell responses by superoptimal numbers of 'nonprofessional' antigen presenting cells. Cellular Immunology 155, 183-194.

Schenk H, Klein M, Erdbrügger W, Dröge W \& Schulze-Osthoff K (1994) Distinct effects of thioredoxin and antioxidants on the activation of NFKB and AP-1. Proceedings of the National Academy of Sciences USA 91, 1672-1676.

Schreck R, Rieber P \& Baeuerle PA (1991) Reactive oxygen intermediates as apparently widely used messengers in the activation of the NFKB transcription factor and HIV-1. EMBO Journal 10, 2247-2258.

Staal FJ, Ela SW, Roederer M, Anderson MT, Herzenberg LA \& Herzenberg LA (1992) Glutathione deficiency and human immunodeficiency virus infection. Lancet 339, 909-912.

Walmsley SL, Winn LM, Harrosin ML, Uetrecht JP \& Wells PG (1997) Oxidative stress and thiol depletion in plasma and peripheral blood lymphocytes from HIV-infected patients: toxicological and pathological implications. AIDS 11, 1689-1697.

Webel DM \& Baker DH (1999) Cystine is the first limiting amino acid for utilization of endogenous amino acids in chicks fed a protein-free diet. Nutrition Research 19, 569-577.

Yoshida A \& Moritoki K (1974) Nitrogen sparing action of methionine and threonine in rats receiving a protein-free diet. Nutrition Reports International 9, 159-168. 